Supporting Information for:

\title{
Using Redox Titrations to Probe the Role of Trivalent Impurity Ions in the Ferromagnetism of Colloidal EuS Nanocrystals
}

\author{
Michael C. De Siena, ${ }^{\dagger}$ Alexander G. Rachkov, ${ }^{\dagger}$ Rachel Fainblat, ${ }^{\dagger \S}$ Derak James,${ }^{\dagger}$ \\ Sidney E. Creutz, ${ }^{\dagger} \perp$ Sarah L. Stoll, ${ }^{{ }^{*}}$ and Daniel R. Gamelin ${ }^{\dagger *}$ \\ ${ }^{\dagger}$ Department of Chemistry, University of Washington, Seattle, WA 98195-1700 \\ ${ }^{\ddagger}$ Department of Chemistry, Box 571227, Georgetown University, Washington, D.C. 20057 \\ *Email: gamelin@chem.washington.edu, sls55@georgetown.edu
}

\section{Additional Data}
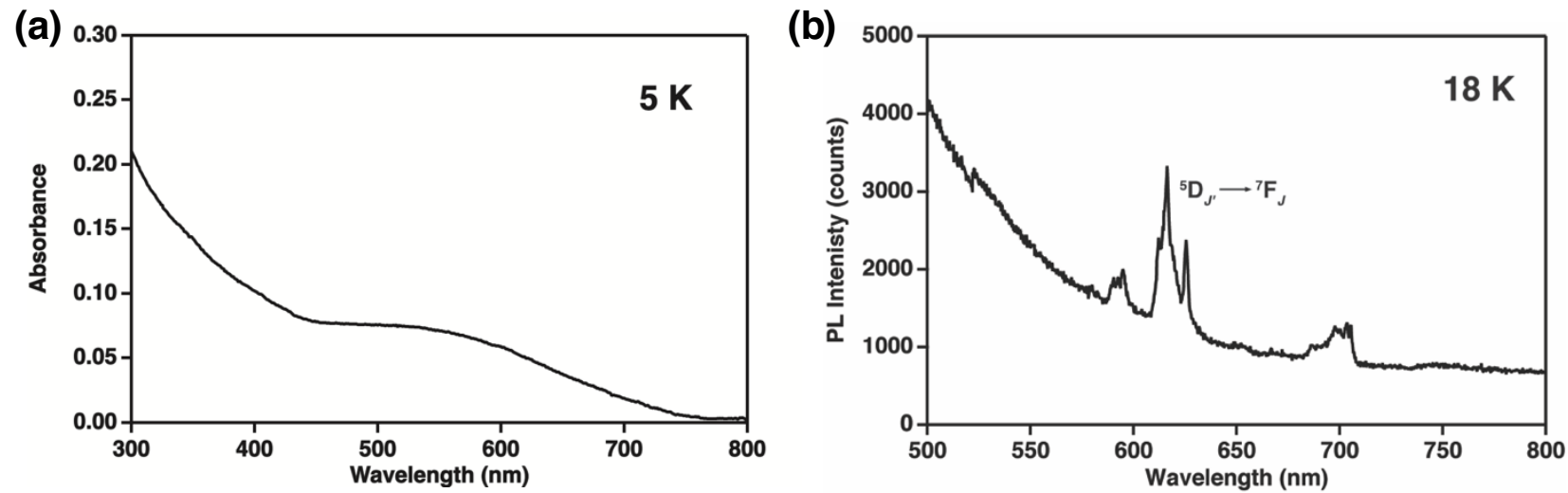

Figure S1. Representative low-temperature (a) absorption and (b) photoluminescence spectra of EuS NCs. The low-temperature $(5 \mathrm{~K})$ absorption spectrum shows no discernible sharp $f-f$ transitions of $\mathrm{Eu}^{3+}$. The photoluminescence spectrum collected at $18 \mathrm{~K}$ shows sharp features (e.g., centered at $\sim 620 \mathrm{~nm}$ ) assigned to the $f-f$ transitions of $\mathrm{Eu}^{3+}$. This result suggests that the $\mathrm{NCs}$ contain $\mathrm{Eu}^{3+}$ defects. These $\mathrm{Eu}^{3+}$ ions are not observed by absorption spectroscopy because of the very low oscillator strengths of the $f-f$ transitions. Notably, there is no evidence of the broadband luminescence that is typical of $\mathrm{EuS}^{1}$ or related $\mathrm{Eu}^{2+}$-containing chalcogenides (e.g., $\mathrm{Eu}^{2+}$-doped $\mathrm{CaS}^{2}$ ). The broad tail of a higher-energy emission is seen and this feature is tentatively assigned to emission from either ligands or NC trap states. 


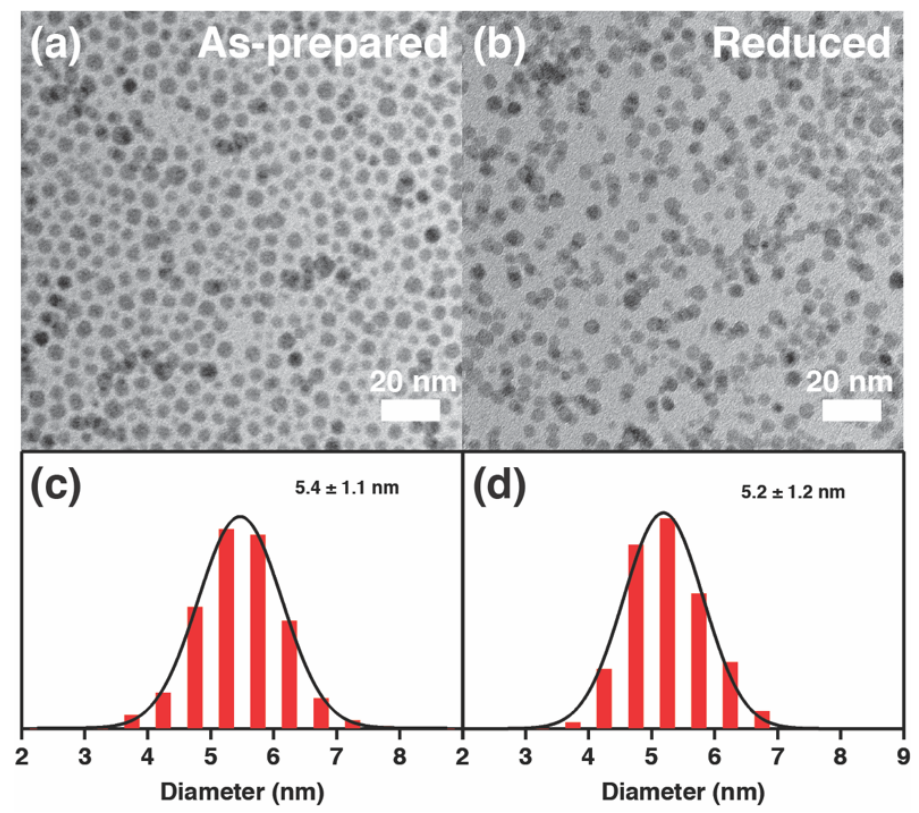

Figure S2. TEM images and corresponding size distributions of EuS NCs before and after reduction with sodium anthracenide. There is no obvious change to the morphology of the EuS NCs after the reduction, as seen in the images for the as-prepared (a) and reduced (b) NCs. Likewise, there is no significant change to the size distribution between the as-prepared (c) and reduced (d) NCs. This result suggests that the reductant is not etching or degrading the NCs in any detectable way. 

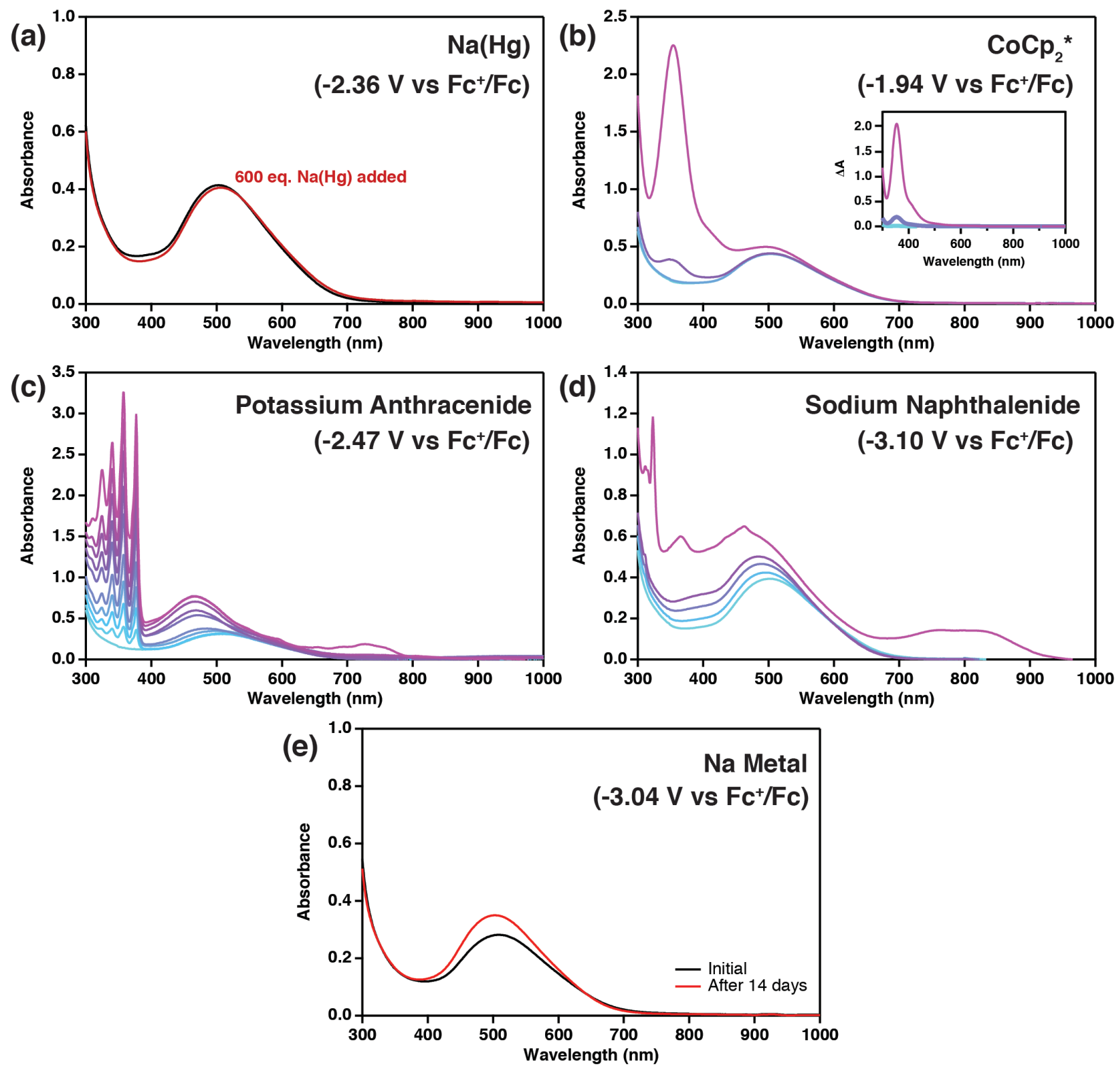

Figure S3. Reduction of EuS NCs with various reductants. The EuS NCs do not show any reduction when the strength of the reductant is insufficient, as seen with both $0.5 \% \mathrm{Na}(\mathrm{Hg})(\mathrm{a})$ and with $\mathrm{CoCp}_{2}{ }^{*}$ (b). The inset shows the change in absorption with added $\mathrm{CoCp}_{2}{ }^{*}$ and is consistent with the absorption of pure $\mathrm{CoCp}_{2}{ }^{*}$, suggesting that the absorption change in the sample is not the result of reduced EuS NCs. In (c), NC reduction is observed with potassium anthracenide. This reductant does not show any significant differences relative to sodium anthracenide (discussed in the main text). Even stronger reductants such as sodium naphthalenide (d) and sodium metal in THF (e) result in the typical reduction behavior of EuS NCs. It is noted that the reduction of EuS NCs by sodium metal is considerably slower that that using the various homogeneous reductants examined here, most likely because of its heterogeneous nature. Strengths of reductants are taken from the literature and given in each panel. $^{3}$ 


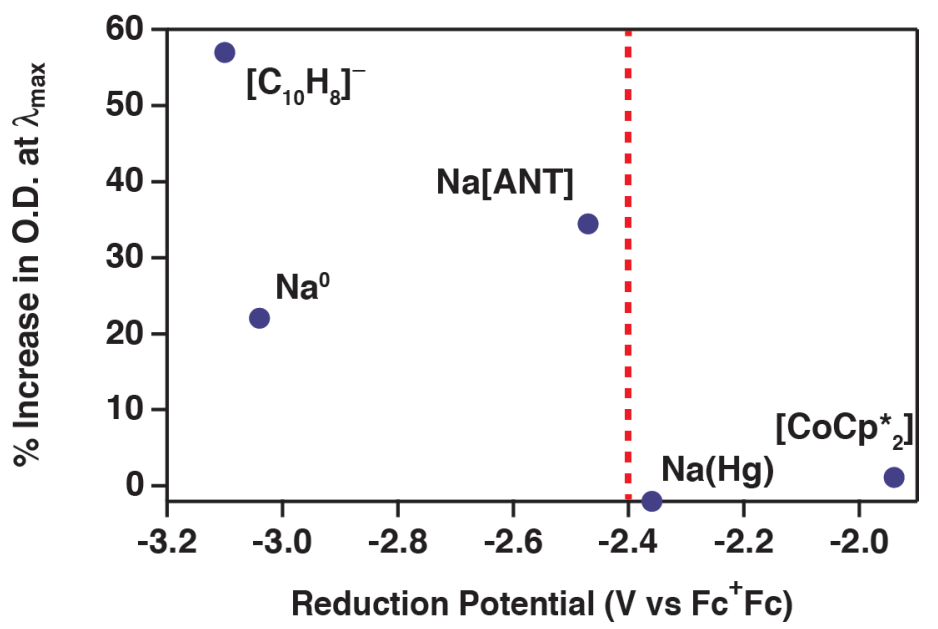

Figure S4. Summary of reduction of EuS NCs with various reductants, plotting the percent increase in the optical density at $\lambda_{\max }$ of the $f-d\left(t_{2 \mathrm{~g}}\right)$ transition $v s$ the standard reduction potentials $s^{3}$ of the reductants. The red vertical line is a guide to the eye indicating the cutoff for reduction of EuS NCs.

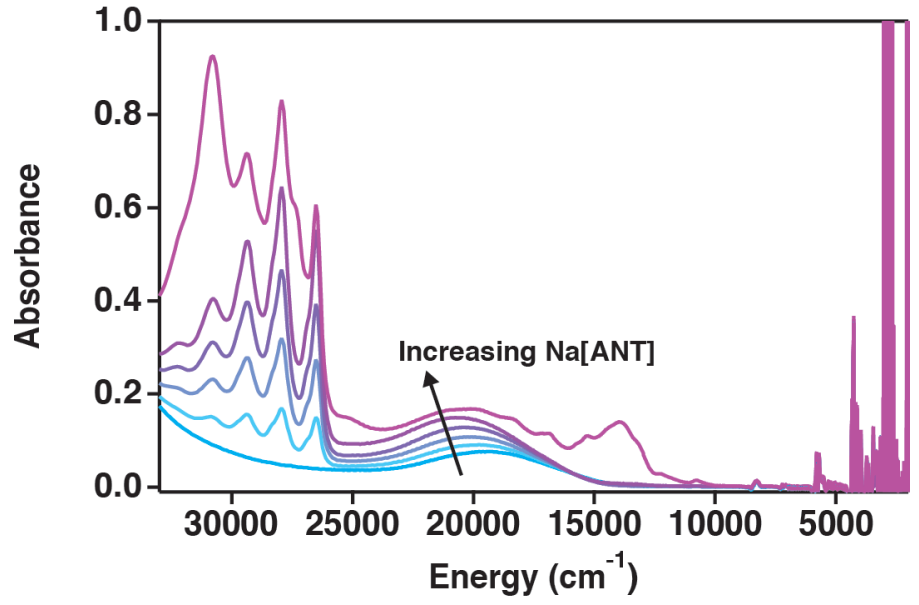

Figure S5. UV-Vis-NIR absorption spectra of EuS NCs during chemical reduction using sodium anthracenide. There is no new NIR intraband or plasmonic band observed during this reduction process. 

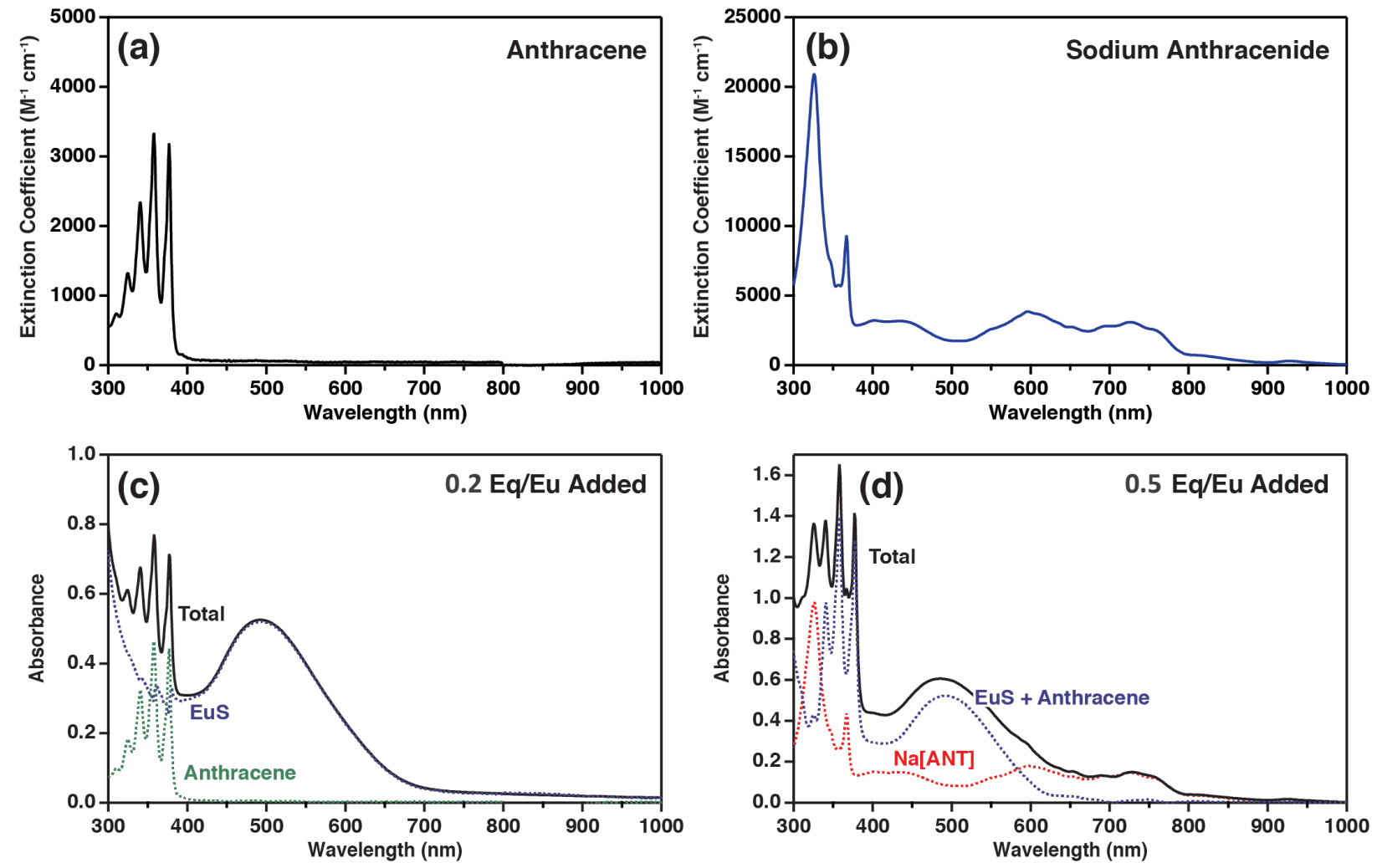

Figure S6. Optical extinction spectra for anthracene (a) and sodium anthracenide (b). The extinction coefficients from these spectra were used for determining the concentrations of these species when calculating the Fermi energy of the system. Panels (c) and (d) show examples of deconvolved absorption spectra. Panel (c) shows the absorption spectrum measured after addition of 0.2 eqivalents of $\mathrm{Na}[\mathrm{ANT}]$, where only anthracene and EuS NCs contribute to the absorption spectrum, and panel (d) shows the absorption spectrum measured at the end point of the titration, where there is also significant absorption from sodium anthracenide. 

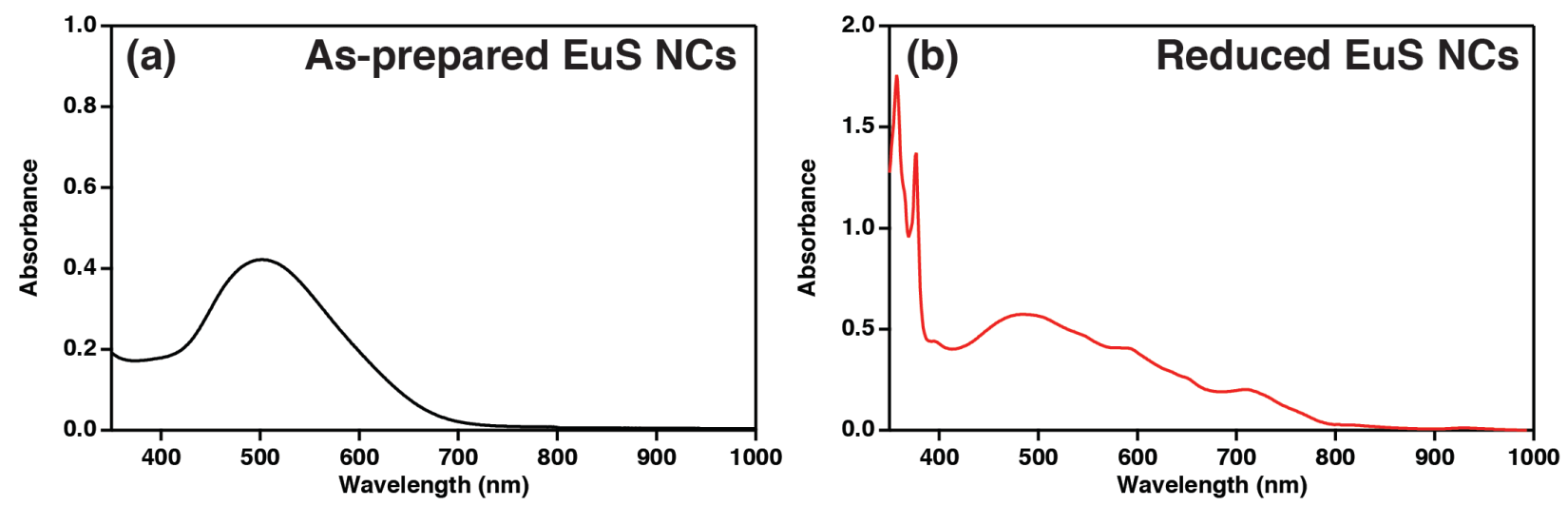

Figure S7. Absorption spectra of the as-prepared (a) and reduced (b) EuS NCs used for the EPR measurements. The reduced NCs show the characteristic increase in the intensity and slight blueshift of the $f-d$ transition (centered at $\sim 490 \mathrm{~nm}$ ) as well as the sodium anthracenide transition (at $750 \mathrm{~nm}$ ). Both samples used for EPR measurements have the same concentration of EuS NCs.

(a)

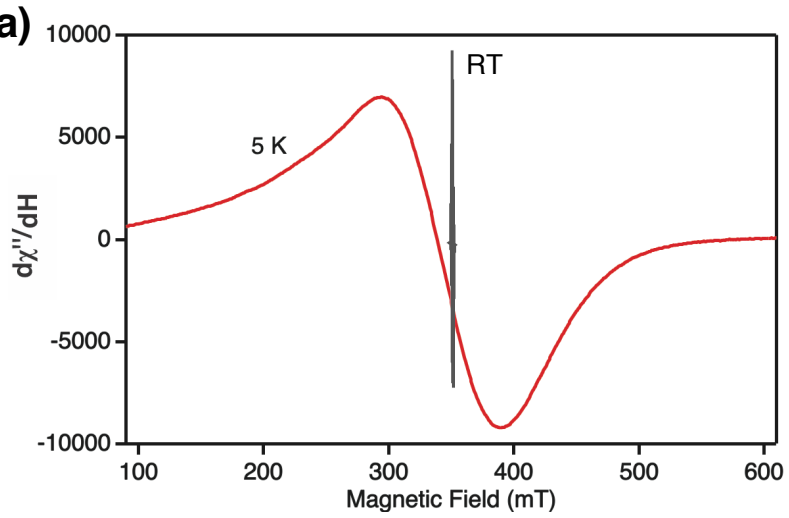

(b)

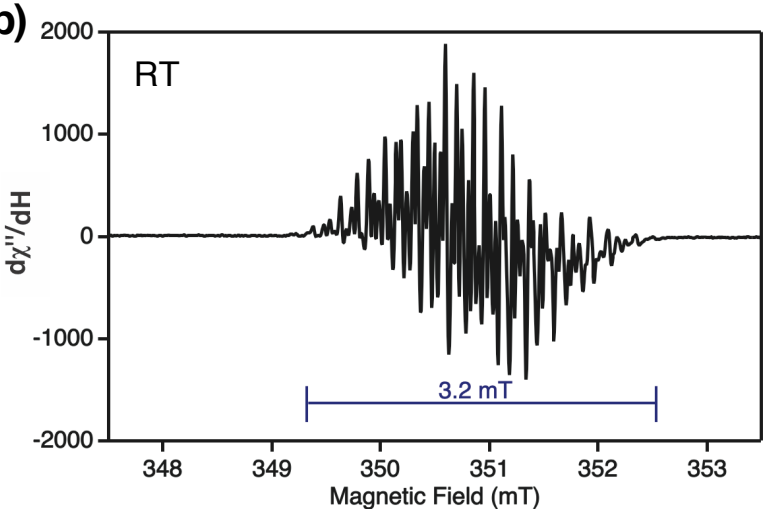

Figure S8. (a) Comparison of the EPR spectra of reduced EuS NCs (5 K) and Na[ANT] (298 K). (b) An expanded view of the $\mathrm{Na}[\mathrm{ANT}]$ resonance at room temperature. The total width of this resonance is $\sim 3.2 \mathrm{mT}$. $n$-propylether is used as the solvent for the $\mathrm{Na}[\mathrm{ANT}]$ spectrum because of solvent dielectric issues in the room-temperature measurements. $\mathrm{No} \mathrm{Na}$ [ANT] signal is observed in the spectrum of the reduced EuS NCs. Up to the equivalence point, $\mathrm{Na}[\mathrm{ANT}]$ is consumed by reaction with the NCs and does not accumulate. Beyond the equivalence point, Na[ANT] is still difficult to observe in the same spectrum because of the large modulation amplitude needed for measuring the EuS NC EPR spectrum. 

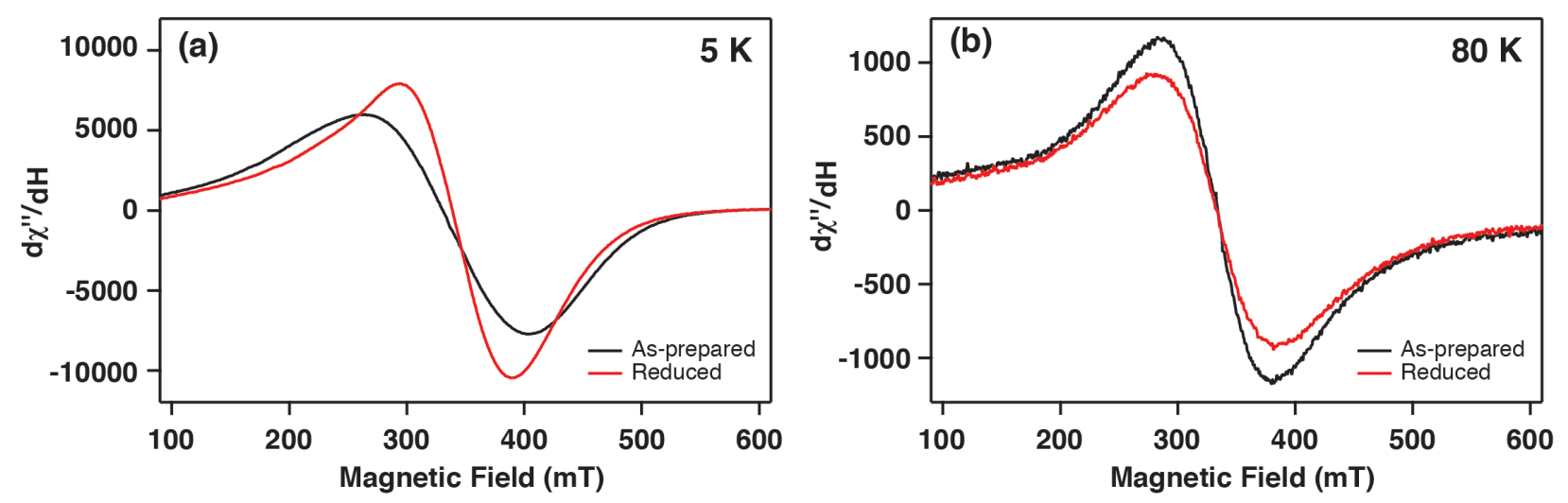

Figure S9. Representative EPR spectra of as-prepared and reduced EuS NCs collected at 5 (a) and $80 \mathrm{~K}(\mathrm{~b})$.

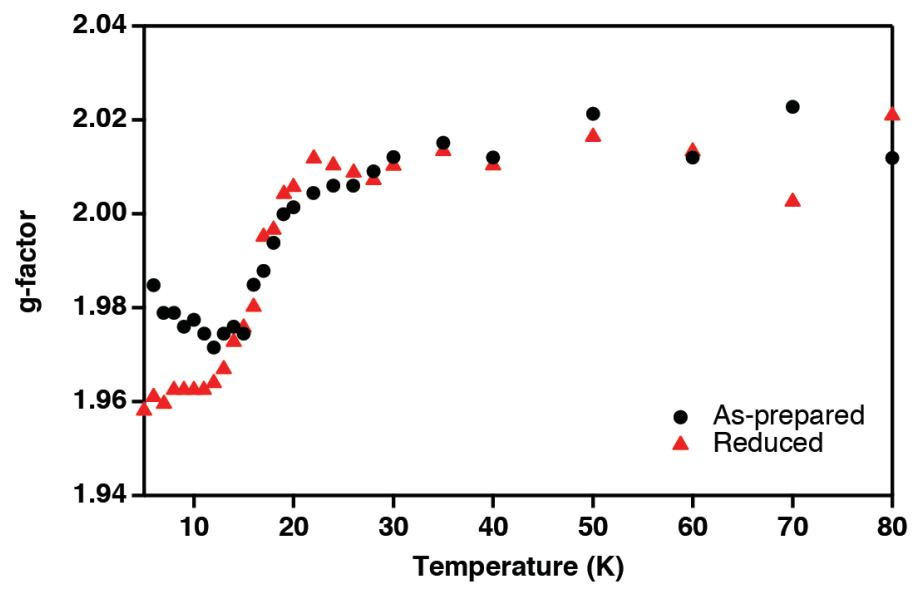

Figure S10. Temperature-dependence of the EPR $g$ factor for as-prepared and reduced EuS NCs. The $g$ factors of both the as-prepared and reduced NCs decrease sharply in the critical region. 
Present address: Department of Chemistry and Biochemistry, University of California, San Diego, La Jolla, CA 92093, USA

${ }^{\S}$ Present address: Werkstoffe der Elektrotechnik and CENIDE, University of Duisburg-Essen, Bismarckstraße 81, 47057 Duisburg, Germany

${ }^{\perp}$ Present address: Department of Chemistry, Mississippi State University, Mississippi State, MS 39762, USA

\section{References}

(1) Chen, W.; Zhang, X.; Huang, Y., Luminescence enhancement of EuS nanoclusters in zeolite. Appl. Phys. Lett. 2000, 76, 2328-2330.

(2) Zhao, Y.; Rabouw, F. T.; van Puffelen, T.; van Walree, C. A.; Gamelin, D. R.; de Mello Donegá, C.; Meijerink, A., Lanthanide-Doped CaS and SrS Luminescent Nanocrystals: A Single-Source Precursor Approach for Doping. J. Amer. Chem. Soc. 2014, 136, 1653316543.

(3) Connelly, N. G.; Geiger, W. E., Chemical Redox Agents for Organometallic Chemistry. Chem. Rev. 1996, 96, 877-910. 